\title{
MQI Based Face Recognition Under Uneven Illumination
}

\author{
Yaoyao Zhang, Jie Tian, Xiaoguang He, and Xin Yang \\ Center for Biometrics and Security Research, Key Laboratory of Complex Systems and \\ Intelligence Science, Institute of Automation, Chinese Academy of Sciences, P.O. Box 2728, \\ Beijing, 100080, P.R. China \\ tian@ieee.org, jie.tian@ia.ac.cn
}

\begin{abstract}
Face recognition has been applied in many fields, while face recognition under uneven illumination is still an open problem. Our approach is based on Morphological Quotient Image (MQI) for illumination normalization, and Dynamic Morphological Quotient Image (DMQI) is proposed to improve the performance. Before applying MQI, singularity noise should be removed, and after MQI operation, an effective scheme is used to wipe off the grainy noise as postprocessing. Weighted normalized correlation is adopted to measure the similarity between two images. Experiments on Yale Face Database B show that the proposed MQI method has a good performance of face recognition under various light conditions. Moreover, its computational cost is very low.
\end{abstract}

Keywords: face recognition, illumination normalization, quotient image, morphological operation.

\section{Introduction}

Face recognition shows its importance nowadays, and more and more fields, such as intelligent human-computer interfaces and e-services, have been benefited from it [1]. Since the common and economical face recognition systems are based on face images captured in the visible light spectrum, the changes of environment light may do harm to the performance of the systems. Due to the uncertainty of light condition and the complexity of models, face recognition under uneven illumination gets more and more attention.

In recent years, many techniques have been proposed to solve such problem, among which quotient image method accounts for an important part. Quotient Image (QI) [2], first proposed by Shashua et al., is a novel means to address the illumination problem. It makes use of illumination invariant information, which is achieved by computing the ratio of a probe image and the linear combination of three known images under different light conditions, but the performance degrades if the main features between the test set and the bootstrap are misaligned. Quotient Illumination Relighting (QIR) [3] method gets the image of subject under canonical light condition by calculating the ratio between an arbitrary image and its quotient illumination. However, QIR relies on the assumption that the illumination of the current subject is 
known in advance, while in most applications, such assumption can not be satisfied. $\mathrm{H}$. Wang et al. brought out the conception of Self-Quotient Image (SQI) [4], and this method aims to estimate the light condition over the image by using Weighted Gaussian filter, but there may be some over-compensation regions when applying SQI. Total Variation based Quotient Image (TVQI) [5], developed by T. Chen et al., is an effective method for face recognition under different illumination. It is based on the estimation of light by solving an optimal problem, which is known as total variation function. However, because of its computational cost, it is not competent for real-time systems.

In this paper, we proposed a new face recognition method based on Morphological Quotient Image (MQI) [6]. It employs mathematical morphological theory and quotient image technique for illumination normalization. Such method obtains good test results on the publicly available face database, and the time consuming is very low. In despite of its low recognition error rates, the face recognition effect still can be further improved by choosing the size of templates for close operation dynamically, and adopting weighted normalized correlation as the measure of similarity. In addition, an effective scheme is brought out to wipe off the noise generated by division operation in the quotient image.

\section{Morphological Quotient Image}

Given an image $I$ under certain light condition, we can represent it as the product of illumination $L$ and the reflectance $R$ [7], and in most cases, there may be some additional noise $N$. Such model can be expressed as follows:

$$
I(i, j)=L(i, j) R(i, j)+N(i, j) .
$$

Where we assume that illumination $L$ is the smooth version of the original image with sharp edges, which shows the variation of the light intensity. Reflectance $R$, i.e. albedo, relies on the surface texture of the object, which is light free and stable for recognition. Additional noise $N$ may do harm to the performance of face recognition algorithms, so it should be removed at first.

The method of Morphological Quotient Image (MQI) uses close operation, a kind of morphological approach, for light estimation. Close operator is a nonlinear operator, which is defined simply as a dilation followed by an erosion with the same structuring element. In general, dilation makes objects to expand or grow in size, while erosion causes objects to shrink. Therefore, with a suitable structuring element, close operation can preserve some particular patterns while attenuating others, and moreover, the original edges will not be blurred. Specially, when dealing with an image under uneven illumination, such morphological technique can be used to get a smooth version, and in such smooth image, the region with the points of the same gray value is believed to share the same light intensity. Fig.1 shows an example.

According to the model referred to above, reflectance $\mathrm{R}$ can be obtained simply as follows: 


$$
R(i, j)=\frac{I(i, j)-N(i, j)}{L(i, j)}=\frac{\operatorname{Denoise}(I)}{\operatorname{Close}(\operatorname{Denoise}(I))} .
$$

We can denoise the image before applying close operation, and then compute the ratio of the gray values pixel by pixel before and after the image is morphological processed. The result is light free, so it can be used to face recognition. DMQI is proposed to dynamically choose the size of templates for close operation. Moreover, division operation may generate some grainy noise, especially in the dark regions, and such noise may do harm to face recognition. A simple and effective scheme is proposed to wipe the noise off as the postprocessing.

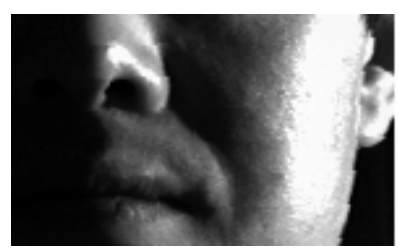

(a)

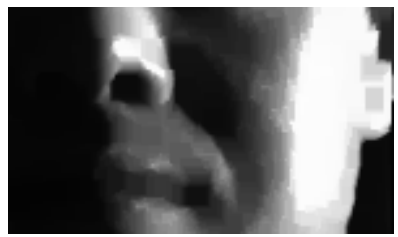

(b)

Fig. 1. (a) The original image under uneven illumination. (b) The processed image after close operation.

\subsection{DMQI}

MQI choose $N^{*} N$ template as the structuring elements, and the size $N$ is the key parameter. With a large template, the close operation would focus on large scale features, but meanwhile has poor performance on local illumination compensation, especially in shadow. On the other hand, if the size is small, it results in good local illumination normalization, but simultaneously misses large scale features. In order to solve the problem, Dynamic Morphological Quotient Image (DMQI) is put forward to make the template size to be adaptive to the given image. The scheme of DMQI can be expressed as follows:

$$
\text { DClose }(i, j)=\left\{\begin{array}{lc}
\operatorname{Close}^{l}(i, j), & \operatorname{Close}^{l}(i, j)>\alpha \cdot \operatorname{Close}^{s}(i, j) \\
\operatorname{Close}^{m}(i, j), & \alpha \cdot \operatorname{Close}^{s}(i, j)>\operatorname{Close}^{l}(i, j)>\beta \cdot \operatorname{Close}^{s}(i, j) . \\
\operatorname{Close}^{s}(i, j), & \beta \cdot \operatorname{Close}^{s}(i, j)>\operatorname{Close}^{l}(i, j)
\end{array}\right.
$$

Where $\alpha$ and $\beta$ are the parameters of the feature scales, while $\alpha>\beta>1.0 . l, m$, and $s$ are the optional sizes of templates, while $l>m>s>1$. If $\operatorname{Close}^{l}(i, j)>\alpha \cdot \operatorname{Close}^{s}(i, j)$, which shows that the result of large size template is very different from that of small one, the area around point $(i, j)$ would be brow, eye, nose, mouth, or the boundary of light intensity changing, where the gray values of the pixels change significantly. 
Therefore, large size template is chosen to keep features. If $\beta \cdot \operatorname{Close}^{s}(i, j)>\operatorname{Clos}^{l}(i, j)$, which indicates that large template and small one have almost the same effect, the point $(i, j)$ is in a smooth region, such as cheek and forehead, and the region is under even illumination. Gray values of the pixels in such areas have little changes. In this case, small size template is a good choice for the close operation.

\subsection{Preprocessing}

Denoising the image before applying MQI is necessary because noise can decrease the performance of the method, especially the singularity noise, which is defined as the points having the value to be the unique maximum or unique minimum of their neighbors' values. The denoising method can be expressed as follows:

Denoise $(I(i, j))=\left\{\begin{array}{cc}\underset{(n, m) \in \text { Meighbor }(i, j)}{\text { Maximum }}(I(n, m)) & \forall(n, m) \in \text { Neighbor }(i, j), I(i, j)>I(n, m) \\ \underset{(n, m) \in \text { Meighimur }(i, j)}{\text { Minimum }}(I(n, m)) & \forall(n, m) \in \text { Neighbor }(i, j), I(i, j)<I(n, m) \\ I(i, j) & \text { otherwise }\end{array}\right.$

Where Neighbor $(i, j)$ means the 8-neighbor set of point $(i, j)$. If the gray value of the current point is larger (smaller) than any of its 8-neighbor points, the point is given the value of maximum (minimum) of the gray values of all its 8 neighbors.

\subsection{Postprocessing}

Morphological Quotient Image method can be used to get the light free information of the objects, but in some regions, especially in shadows, grainy noise is generated with the operation. This is due to the division operation in the MQI model. Since the gray values of points in the dark region are very small compared to the points in the light region, division operation amplifies the original small gray value changes in the dark region, and such small changes are not the robust feature of the objects. Fig. 2 illustrates the generation of such noise. Image (d) shows that the gray values of the pixels on the line in image (c) fluctuate more significantly in the left part than that in the right part, and it is for the reason that the left part of original image is darker than the right part.

Removing the grainy noise is necessary since the noise does harm to face recognition. Here, by analyzing the main traits of such kind of noise, as well as the difference between the noise and useful texture information, a simple scheme is cast to wipe off the noise effectively and efficiently. For each point in the image, and all its 8-neighbor elements, we sort the 9 points in order according to their gray values, and check the value difference between neighbor ones. The point is considered to be noise if the values of neighbor differences are all small enough (except that all the neighbor differences are zero), and thus the point is given the value of the maximum of the 9 points. Otherwise, the point is thought to be the useful information and then keep its original value. 


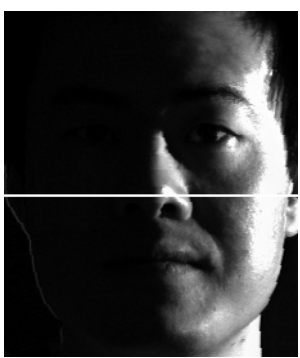

(a)

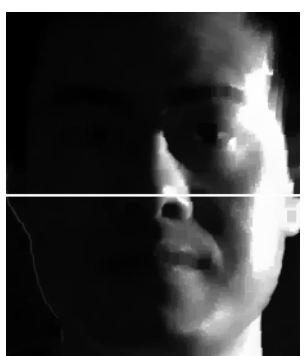

(b)

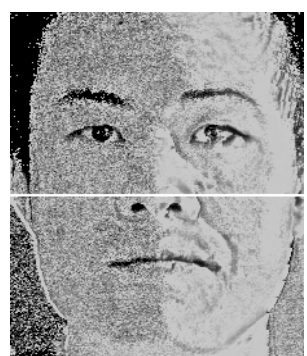

(c)

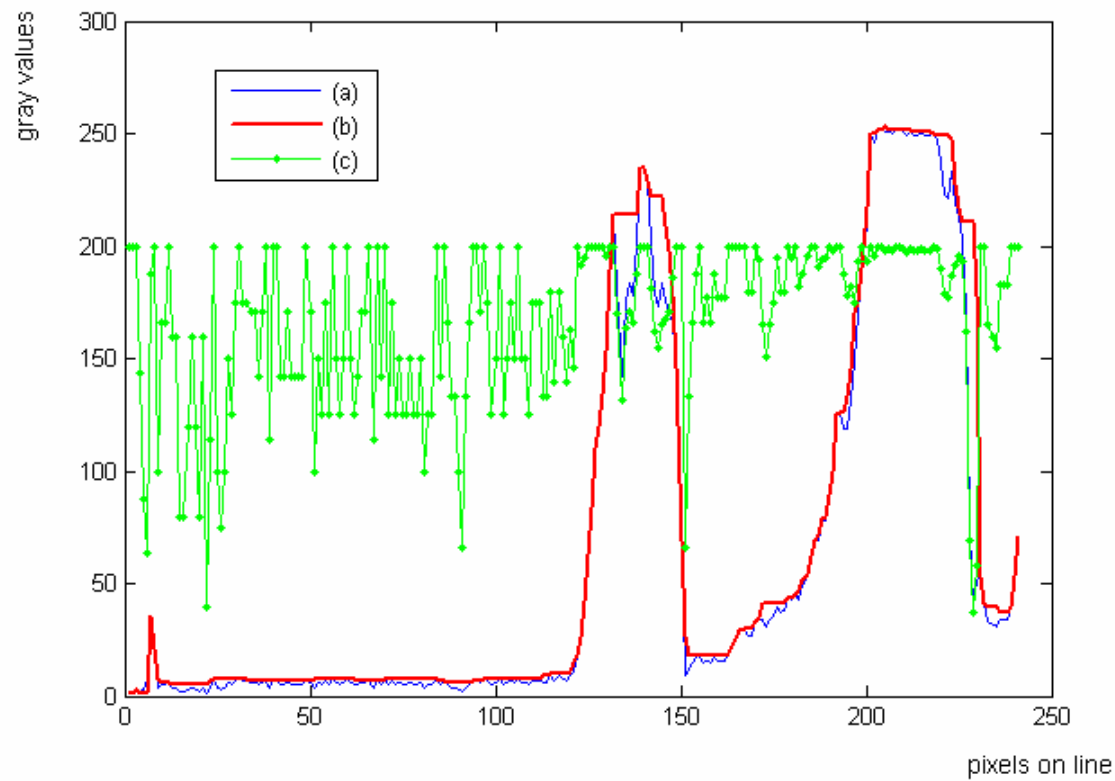

(d)

Fig. 2. (a) The original image. (b) The processed image after MQI operation. (c) The result of quotient image. All the three images have a line on them at the same position. (d) Changes of gray values of the pixels on the line of each image. Blue one refers to (a); red line refers to (b); green one refers to image (c).

\section{Experiments}

Face image is normalized according to the coordinates of eyes, and cropped to proper size in order that only the face region is used for recognition. It is first preprocessed to remove the singularity noise, and then morphological close operation is applied to the image. The ratio of the image before and after close operation is computed to get the light free information, and the grainy noise is wiped off as postprocessing. Face recognition is performed after face images are processed by MQI method. 
Usually there are a large number of test images to be classified, while only a few images can be used as the training set; therefore, many sophisticated classifiers are not necessary, and nearest neighbor classifier is chosen to be used. Particularly, we can here have only one image in the gallery for each class. The distance between two images can be measured in various ways, for example, Euclidean Distance is the most common one, and here, we measure the dissimilarity of two images with normalized correlation, i.e. cosine of the angle between the two vectors, which is proved to be effective in this case. The similarity between two images can be computed as follows:

$$
\Phi\left(I_{i}, I_{t}\right)=\cos \left(\angle\left\langle I_{i}, I_{t}\right\rangle\right)=\frac{I_{i} \cdot I_{t}}{\left\|I_{i} \mid\right\| I_{t} \|} .
$$

Furthermore, not every local region of face plays the equal role on the effect of face recognition, and generally, some particular parts, such as brow, eye, nose and mouth, have most of the key features for identification. In addition, the face images to be classified have been normalized according to the coordinates of two eyes. That different local part of face has different weights value is more effective when measuring the similarity of two images. The weights are selected through experiment, not utilizing an actual optimization procedure, and thus they are probably not optimal. Fig. 3 shows an example. Compared to the method with equal weights, we get an improvement in the recognition rate.

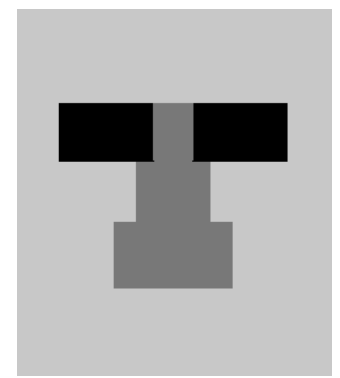

Fig. 3. An example of weight set over the face image. The darker the region is, the higher the weight value is.

The MQI method is tested on Yale Face database B, which includes 10 subjects, each with 64 frontal faces under different light conditions. In the 640 face images, there are no variations of pose, expression, age and so on. Those images are aligned according to the coordinates of eyes, and are cropped to size $281 * 241$ in order that only the face region is used. All the 640 images are divided into five subsets according to the angle between the light source direction and the camera axis: Subset $1\left(0^{\circ}\right.$ to $\left.12^{\circ}\right)$, Subset $2\left(13^{\circ}\right.$ to $\left.25^{\circ}\right)$, Subset $3\left(26^{\circ}\right.$ to $\left.50^{\circ}\right)$, Subset $4\left(51^{\circ}\right.$ to $77^{\circ}$ ), and Subset 5 (above $78^{\circ}$ ), and each subset respectively has $70,120,120,140$, 190 face images. 7 corrupted images are discarded [8], and therefore there are totally 633 images left: Subset 1 to 5 respectively has 70, 118, 118, 138 and 189 images. 


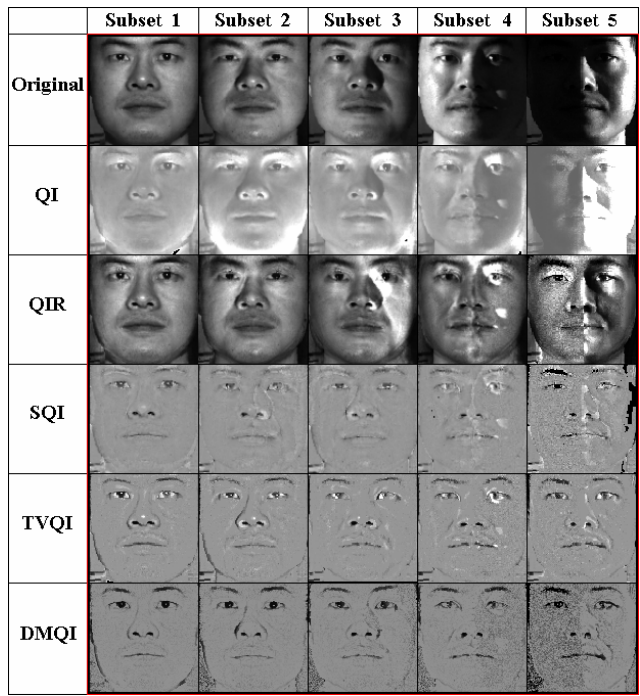

Fig. 4. Contrast of processed images after various quotient image operation

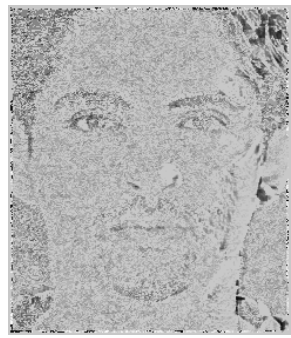

(a)

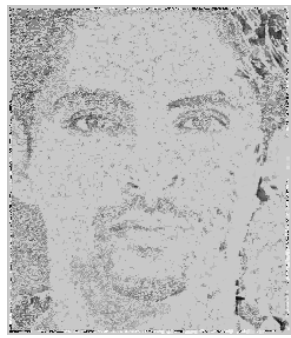

(b)

Fig. 5. (a) Face image with grainy noise. (b) Face image after being processed.

One of the advantages of MQI method is that only one image needed in the train set for each class, and here we choose the face images under frontal illumination as the templates, the other 623 images as the test samples. We first process the total 10 register images with MQI method, and then save them as templates. For each of the 623 test images, after being processed in the same way, we measure the similarities between the test image and each of the saved templates, and then decide the identity of test sample according to the nearest neighbor rule. Through experiment, The template size to be 7 is a proper selection, and in the case of adopting DMQI scheme, when parameters $\alpha=1.8, \beta=1.35$, size of template $s=5, m=7, l=9$, the performance of illumination normalization is pretty good. Fig. 4 shows the contrast of face images processed by several kinds of quotient methods, and these images are selected from the 5 subsets of Yale Face database respectively. We can see that the results of QI and QIR are not so competent as the other three, and in SQI, there exists some over-compensation regions, which shows additional dark blocks in appearance. TVQI and DMQI both have good performance visually on illumination normalization, 
while there seems to be more grainy noise generated by DMQI. Such grainy noise can be removed using the method referred to above as post processing, and experiments proved that the performance is quite good. Fig. 5 gives an example. Most of the noise is wiped off, and at the same time useful information are kept.

Table 1. RER Comparisons of various methods on Yale Face database B

\begin{tabular}{|c|c|c|c|c|c|}
\hline \multirow{2}{*}{ Methods } & \multicolumn{5}{|c|}{ Recognition Error Rate (\%) vs. Illumination } \\
\cline { 2 - 6 } & $\begin{array}{c}\text { Subset } \\
1\end{array}$ & $\begin{array}{c}\text { Subset } \\
2\end{array}$ & $\begin{array}{c}\text { Subset } \\
3\end{array}$ & $\begin{array}{c}\text { Subset } \\
4\end{array}$ & $\begin{array}{c}\text { Subset } \\
5\end{array}$ \\
\hline Correlation[8] & N/A & 0.0 & 23.3 & 73.6 & N/A \\
\hline $\begin{array}{c}\text { Eigenface w/o } \\
\text { 1st3 [8] }\end{array}$ & N/A & 0.0 & 19.2 & 66.4 & N/A \\
\hline $\begin{array}{c}\text { Linear Subspace } \\
\text { [8] }\end{array}$ & N/A & 0.0 & 0.0 & 15.0 & N/A \\
\hline Cones Cast [9] & N/A & 0.0 & 0.0 & 10.0 & 37.0 \\
\hline QI & 0.0 & 1.7 & 38.1 & 65.9 & 76.7 \\
\hline QIR & 0.0 & 0.0 & 0.0 & 9.4 & 21.2 \\
\hline SQI & 0.0 & 0.0 & 0.0 & 3.6 & 2.1 \\
\hline $\begin{array}{c}\text { Relative } \\
\text { Difference }\end{array}$ & 0.0 & 0.0 & 0.0 & 0.0 & 12.0 \\
\hline Space[10] & 0.0 & 0.0 & 0.0 & 0.0 & 1.6 \\
\hline MQI & 0.0 & 0.0 & 0.0 & 0.0 & 1.1 \\
\hline DMQI & 0.0 & 0.0 & 0.0 & 0.0 & 0.5 \\
\hline $\begin{array}{c}\text { DMQI + weighted } \\
\text { normalized } \\
\text { correlation }\end{array}$ & 0.0 & 0.0 & 0.0 & 0.0 & 0.0 \\
\hline TVQI & & & & \\
\hline
\end{tabular}

Table 2. Comparison of computational complexity

\begin{tabular}{|c|c|c|c|}
\hline & \multicolumn{3}{|c|}{ Computational complexity comparison } \\
\hline Methods & MQI & DMQI & TVQI \\
\hline $\begin{array}{c}\text { Average processing time per image } \\
\text { (Second) }\end{array}$ & 0.09 & 0.21 & 12.89 \\
\hline \begin{tabular}{c} 
Computational complexity \\
\hline
\end{tabular} & $O(n)$ & $O(3 \cdot n)$ & $O\left(l \cdot n^{3 / 2}\right)$ \\
\hline
\end{tabular}

Table 1 lists the Recognition Error Rates (RER) of different kinds of methods, including the QI schemes referred to above, and some classical techniques as well. It shows that Subset 5 gets the highest RER of all the subsets almost for all the methods, which is due to the acutest variation of the light condition, and therefore, it is most difficult to compensate illumination for the images. MQI outperforms most of the existing methods, and when choosing the size of template for close operation dynamically, DMQI gets lower RER. Moreover, the recognition performance improved when weighted normalized correlation is adopted to measure the similarity between two images. 
RER of TVQI is lower than that of DMQI, but TVQI method is realized by solving an optimal problem, which needs a number of iterations to find the optimal solution. In other words, the time and resource consuming of TVQI is expensive, while MQI technique would be much faster, and is more competent to be used in real-time application. Table 2 shows the comparison of the computational complexity of MQI, DMQI and TVQI, where $n$ is the pixel number of the whole image, and $l$ is the time needed for each iteration for TVQI. Such data is calculated with the mean of the 633 images, and the evaluation is conducted on Dell 4700 with Pentium 4 CPU 2.80GHZ.

\section{Conclusion}

This paper is based on MQI, a new proposed method for illumination normalization. Morphological close operation and quotient computation are used to get light free information. The test on Yale Face Database B shows that it is very effective and efficient, and lower RER can be obtained when adopting DMQI and utilizing weighted nearest neighbor classifier. Moreover, an effective scheme is used to remove the noise generated by MQI as postprocessing.

\section{References}

1. Zhao, W., Chellappa, R., Rosenfeld, A., Phillips, P.: Face recognition: A literature survey. UMD CfAR Technical Report, CAR-TR-948 (2000)

2. Shashua, A., Riklin-Raviv, T.: The quotient image: Classbased re-rendering and recognition with varying illuminations. IEEE Transactions on Pattern Analysis and Machine Intelligence 23(2), 129-139 (2001)

3. Shan, S., Gao, W., Cao, B., Zhao, D.: Illumination normalization for robust face recognition against varying lighting conditions. In: Proc. of IEEE Workshop on AMFG, pp. 157-164. IEEE Computer Society Press, Los Alamitos (2003)

4. Wang, H., Li, S.Z., Wang, Y.: Generalized quotient image. In: IEEE International Conference on Computer Vision and Pattern Recognition. IEEE Computer Society Press, Los Alamitos (2004)

5. Chen, T., Yin, W., Zhou, X., Comaniciu, D., Huang, T.: Illumination Normalization for Face Recognition and Uneven Background Correction Using Total Variation Based Image Models. In: IEEE Computer Society Conference on Computer Vision and Pattern Recognition, vol. 2, pp. 532-539 (2005)

6. He, X., Jie, T., Wu, L., Zhang, Y., Yang, X.: Illumination Normalization with Morphological Quotient Image. Journal of Software 18(9) (2007)

7. Gross, R., Brajovie, V.: An Image Preprocessing Algorithm for Illumination Invariant Face Recognition. In: 4th International Conference on Audio and Video Based Biometric Person Authentication, pp. 10-18 (2003)

8. Georghiades, A., Belhumeur, P., Kriegman, D.: From Few to Many: Illumination Cone Models for Face Recognition under Variable Lighting and Pose. IEEE Trans. on Pattern Analysis and Machine Intelligence 23(6), 630-660 (2001)

9. Georghiades, A., Kriegman, D., Belhumeur, P.: Illumination Cones for Recognition under Variable Lighting: Faces. In: IEEE Computer Society Conference on Computer Vision and Pattern Recognition. IEEE Computer Society Press, Los Alamitos (1998)

10. He, X., Tian, J., He, Y., Yang, X.: Face Recognition with Relative Difference Space and SVM. In: Proce. of the 18th International Conference of Pattern Recognition, HongKong (August 2006) 